\title{
DEPURACIÓN, REUTILIZACIÓN Y ABASTECIMIENTO HÍDRICO EN EL INTERIOR SEPTENTRIONAL DE ALICANTE ${ }^{1}$
}

\author{
Jordi Tormo i Santonja \\ Grupo Interdisciplinario de Estudios Críticos y de América Latina (GIECRYAL) \\ Departamento de Geografía Humana \\ Universidad de Alicante
}

\section{RESUMEN}

Las comarcas de l'Alcoià, la Foia de Castalla y el Comtat agrupan un total de 36 municipios. Se trata de un área en la que las infraestructuras de tratamiento, depuración y reutilización de las aguas residuales están reguladas por la administración pública valenciana de forma coordinada con la gestión realizada por los municipios y las mancomunidades. Durante los últimos años, se han desarrollado en este espacio diversas actuaciones que han permitido mejorar el abastecimiento a los municipios urbanos y rurales y a las zonas industriales y que pueden considerarse de referencia dentro del ámbito alicantino.

La puesta en marcha de programas para la reconversión de las políticas hídricas tradicionales hacia prácticas más ahorrativas y eficaces es una de las principales estrategias que deben aplicarse con el objetivo de mejorar el abastecimiento de agua potable a los municipios y las industrias mediante la depuración y la reutilización de las aguas residuales.

Palabras clave: Depuración, Reutilización, Abastecimiento, Agua Residual.

\begin{abstract}
The districts of l'Alcoià, la Foia de Castalla and el Comtat contain a total of 36 towns and villages. This is an area in which the infrastructures for reclaiming and reusing wastewater are regulated by the regional authorities, and managed by the local and district authorities. In recent years, various initiatives have led to improvements in the water supply to both urban and rural populations and industrial areas; these initiatives are leading the way in solutions to water supply in the province of Alicante.
\end{abstract}

1 Este artículo se enmarca en la tesis doctoral 'Caracterización socioeconómica, políticas y proyectos para el desarrollo de la comarca funcional de l'Alcoià, el Comtat y la Foia de Castalla. Una apuesta por la planificación estratégica territorial' que ha sido realizada gracias a la concesión de una Ayuda a la Investigación (Convocatoria 2007) por parte del Instituto Alicantino de Cultura Juan Gil-Albert (Excma. Diputación de Alicante). 
Implementing programmes designed to move traditional water policies towards more cost-effective and energy-efficient practices is one of the main strategies needed to improve the supply of drinking water to local populations and industry through the effective management of reclaimed and re-used wastewater.

Key words: Reclaimed water, re-used water, supply, wastewater.

\section{Caracterización básica del interior alicantino}

Las comarcas valencianas de l'Alcoià, la Foia de Castalla y el Comtat ${ }^{2}$ se ubican en el interior de la provincia de Alicante y conforman una de las zonas más abruptas del espacio valenciano. Es por lo que parte de este espacio, especialmente los municipios de montaña

FIgURA 1. Mapa de delimitación comarcal y municipal, 2008

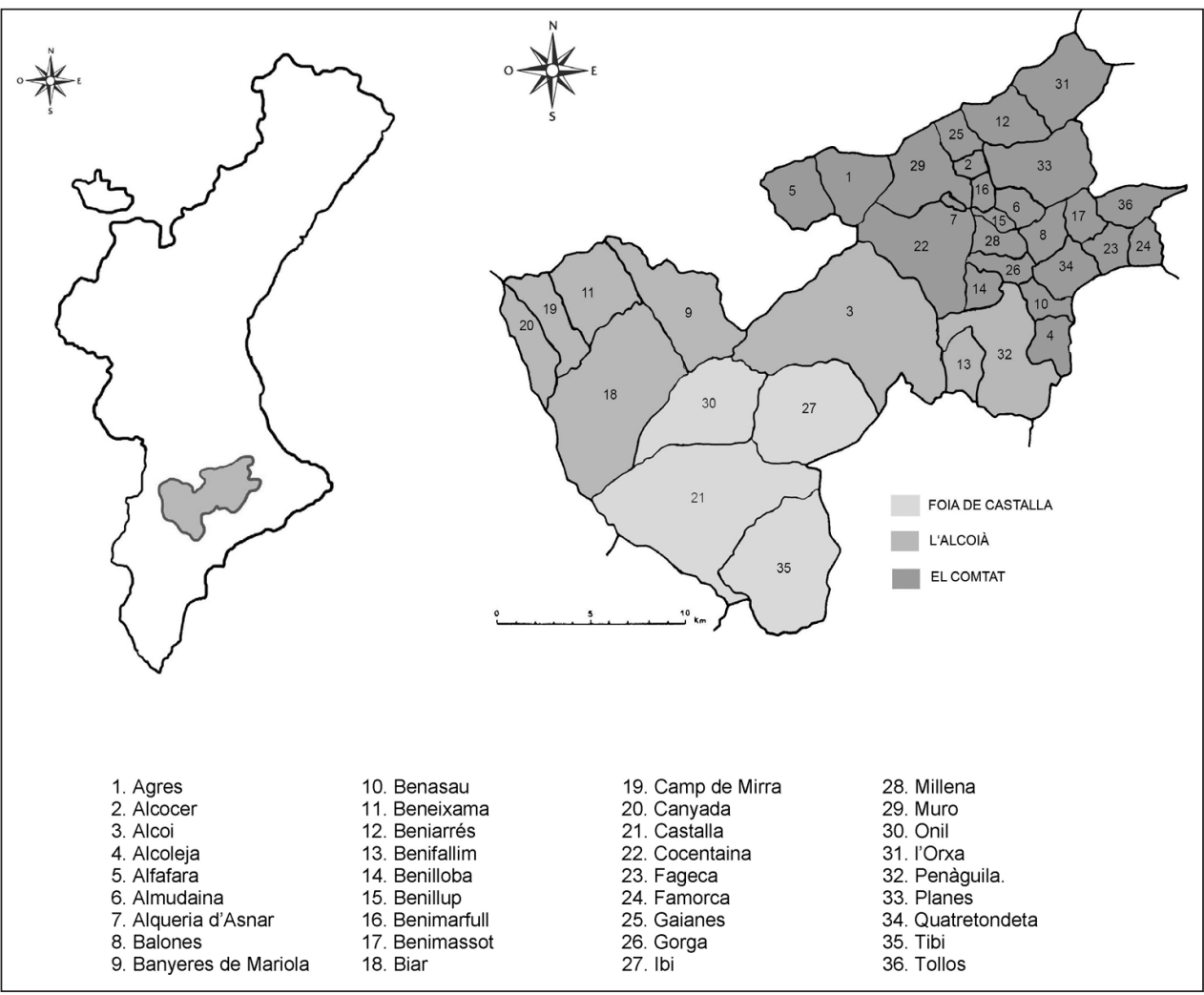

Elaboración propia (2008).

2 La demarcación comarcal utilizada en este trabajo se ha realizado tomando como referencia las propuestas planteadas por F. Mateu (1933), M. Sanchís (1966), E. Beüt (1966), J. Soler (1966), Promocions Culturals (1970) y P. Pérez (1978). 
de las comarcas de l'Alcoià y el Comtat, recibe el nombre de Montaña de Alicante. La mayor parte de los municipios de este espacio geográfico se sitúan a una altitud elevada, destacando Ibi (816 m), Banyeres de Mariola (816 m), Tollos (773 m) y Agres (772 m). Se trata de un área integrada en el Sistema Bético valenciano.

Según los datos del Padrón Municipal de Habitantes 2006, la población total de este espacio se sitúa en 144.551 habitantes, lo que representa un porcentaje del 3,01\% respecto del total de la población valenciana. La comarca más poblada es l'Alcoià con 75.349 vecinos, seguida de la Foia de Castalla (41.729 vecinos) y el Comtat (27.473 vecinos). El principal centro urbano es Alcoi con 60.590 habitantes.

Las comarcas de l'Alcoià, la Foia de Castalla y el Comtat constituyen un área de especialización industrial en la que destacan las actividades del textil-hogar y la confección, el juguete, la transformación del plástico y el caucho, la madera y el mueble y el papel y la edición gráfica. En concreto, según los datos de la Tesorería General de la Seguridad Social, el $40,81 \%$ del empleo se enmarca en el sector industrial, mientras que este porcentaje es tan sólo del $17,31 \%$ en el conjunto valenciano.

\section{La depuración del agua residual en las comarcas de L'Alcoià, La Foia de Castalla y El Comtat}

\subsection{La importancia de la depuración y la reutilización de las aguas residuales}

Según Naredo (2006), durante los últimos años se han presentado dos políticas diferentes de gestión del agua que cuentan con dos enfoques distintos. La primera de ellas, es la promoción de obras hidráulicas desde un enfoque técnico como son los embalses, los trasvases, las desaladoras y los bombeos, entre otras, mientras que por otro lado existe una apuesta por las políticas de agua desde un enfoque socioeconómico que se centran en la implementación de programas de reconversión hacia usos más ahorrativos y eficientes y hacia la flexibilización de los regímenes de concesión como son los bancos de agua, entre otros. En su opinión, «la segunda opción es más económica, más sostenible, pero políticamente más laboriosa. La primera es más despilfarradora, más insostenible, pero políticamente fácil de implementar» (p. 27).

Las aguas residuales urbanas son las que proceden de la actividad humana y no cumplen los requisitos mínimos sanitarios exigibles para el consumo humano, su función natural o su utilización en una serie de usos de diversa tipología. Se trata de aguas que tienen en su composición residuos sólidos, líquidos y gaseosos procedentes de los desechos domésticos, de la escorrentía superficial y de los arrastres de las lluvias, de la actividad industrial y de las infiltraciones, entre otros. Según Hernández (2007), las aguas residuales son los desechos generados tras la utilización del agua por parte del hombre para su actividad y su confort.

La depuración de las aguas residuales se realiza a partir de la aplicación de una serie de tratamientos terciarios con el objeto de reducir los residuos en suspensión. Entre estos procesos destaca la clarificación, la filtración granular, la cloración, la ozonización, los rayos ultravioletas y la filtración por membranas (Pérez Talavera, 2006). Según Bao (2001), las tecnologías avanzadas para el tratamiento de las aguas residuales procedentes de las industrias se pueden clasificar en dos grupos:

- Tecnologías para la reducción y/o eliminación de la contaminación en un único proceso (oxidación, empleo de fitorreactores y tratamientos térmicos o físicos, entre otros). 
- Tecnologías para la reducción y/o eliminación de la contaminación fundamentadas en procesos de separación y tratamientos adicionales (disoluciones, procesos de membranas, extracciones, absorciones y aplicaciones de cambiadores de ión, entre otros).

\subsection{La depuración de las aguas residuales}

2.2.1. Tipología de las infraestructuras para el tratamiento del agua residual

La Conselleria de Medi Ambient, Aigua, Urbanisme i Habitatge es el órgano de la administración pública valenciana que gestiona los servicios de abastecimiento y saneamiento de agua, principalmente a través del organismo público Entitat Pública de Sanejament d'Aigües Residuals (EPSAR). Esta entidad gestiona y mantiene las siguientes instalaciones en el espacio valenciano:

- Estaciones de depuración de aguas residuales (EDAR). Son infraestructuras para la recepción de aguas residuales provenientes de los municipios y las áreas industriales que tras una serie de procesos y tratamientos purifican el agua para su posterior vertido a los cursos fluviales o para su posterior reutilización en los municipios y áreas industriales emisoras.

- Estaciones de tratamiento de aguas potables (ETAP). Son infraestructuras para el abastecimiento supramunicipal de aguas potables a varias localidades.

- Plantas de compostaje. Son infraestructuras para valorar y fomentar la reutilización de los biosólidos generados en las EDAR para la actividad agraria. Su objeto es obtener un producto de valor superior a los lodos de origen.

FiguRA 2. Evolución de las instalaciones para la depuración y del volumen de agua tratada.

Comunidad Valenciana, 2000-2006 (Unidades / $\mathrm{hm}^{3}$ )

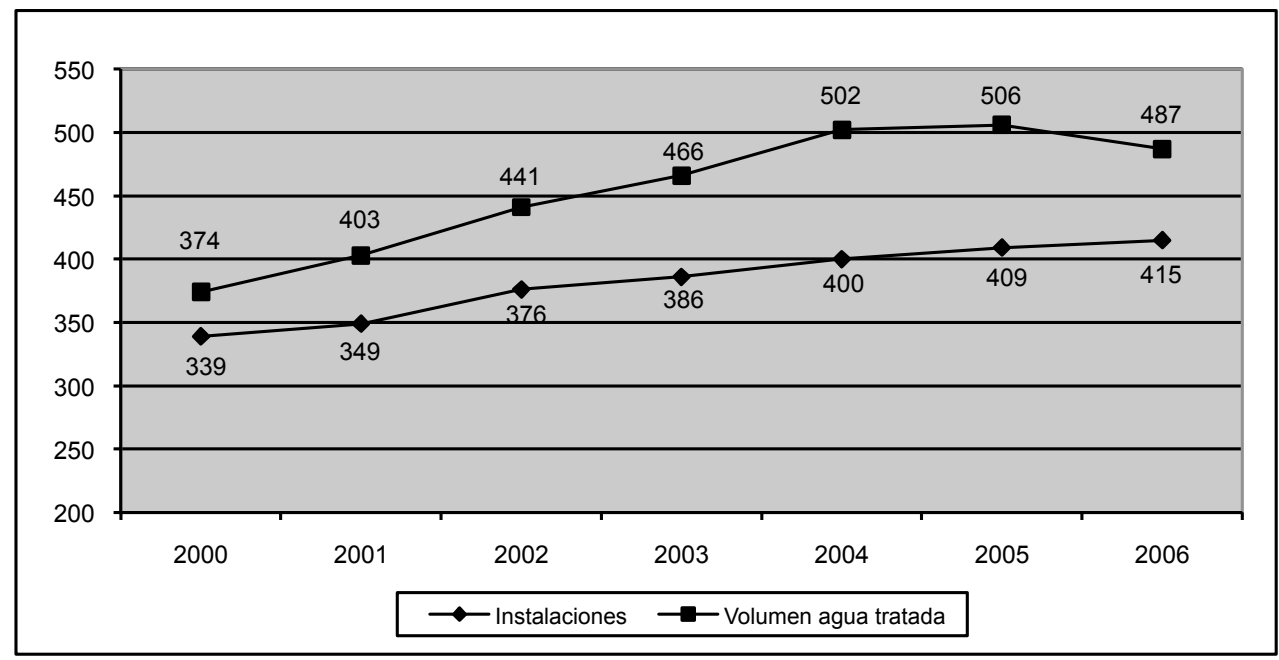

FUENTE: EPSAR (2008). Elaboración propia. 
- Secados térmicos. Son infraestructuras para el secado de los lodos y fangos generados en las EDAR a través de un sistema de inyección de energía térmica.

- Emisarios submarinos. Son infraestructuras para el vertido controlado de aguas depuradas al mar que están sujetas a procesos de vigilancia continua para asegurar la calidad de las aguas de baño.

La evolución de los indicadores de depuración de aguas residuales en el espacio valenciano ha seguido una tendencia positiva durante los últimos años. El número total de instalaciones para la depuración ha incrementado el 22,42\% entre 2000 y 2006, pasando de 339 instalaciones en 2000 a 415 en 2006.

Por otro lado, la evolución del volumen de agua tratada en las instalaciones de depuración también ha seguido una tendencia positiva. En concreto, el volumen de agua tratada ha aumentado el $30,21 \%$ entre 2000 y 2006, pasando de $374 \mathrm{hm}^{3}$ en 2000 a $487 \mathrm{Hm}^{3}$ en 2006. Ahora bien, como se puede observar en la Figura 2, el año 2006 ha registrado un ligero descenso en el volumen de agua depurada.

\subsubsection{Las estaciones de depuración de aguas residuales y las plantas de secado térmico}

Los municipios de las comarcas de l'Alcoià, la Foia de Castalla y el Comtat cuentan con los servicios de 30 estaciones de depuración de aguas residuales EDAR y dos plantas de secado térmico con las que se consigue depurar un caudal de $56.204 \mathrm{~m}^{3} /$ día, según los datos de EPSAR (2008). La comarca con mayor volumen diario de depuración de agua es l'Alcoià $\left(28.275 \mathrm{~m}^{3} /\right.$ día $)$, seguida del Comtat $\left(17.749 \mathrm{~m}^{3} /\right.$ día $)$ y de la Foia de Castalla (10.180 m³/día).

La mayor parte de los municipios disponen de una estación EDAR en su término, si bien algunas de ellas prestan servicio a varios municipios a la vez. En concreto, la EDAR de Font de la Pedra (Muro) depura las aguas provenientes de los términos de Alqueria d'Asnar, Cocentaina y Muro, la EDAR de Foia de Castalla (Castalla) trata las aguas derivadas de los municipios de Castalla y Onil y la EDAR Villena (Villena), que no se encuentra ubicada en las comarcas de l'Alcoià, la Foia de Castalla ni el Comtat, sino en la comarca Alt Vinalopó, depura las aguas procedentes de los municipios de Beneixama, Camp de Mirra, Canyada y Villena.

Las estaciones con mayor capacidad para la depuración de aguas residuales son las de Alcoi (24.000 m³/día), Muro (15.000 m³/día), Villena (8.000 m³/día), Ibi $\left(6.000 \mathrm{~m}^{3} /\right.$ día $)$ y Castalla (4.000 m³/día). Estas cinco estaciones EDAR cuentan con dos líneas de proceso y tratamiento para la depuración del agua residual procedentes de los municipios y las industrias. Son la línea de agua y línea de fango.

La EDAR Els Algars (Alcoi) desarrolla un tratamiento biológico mediante fangos activados y un tratamiento físico-químico mediante la Línea de agua. Este proceso consta de las fases de pretratamiento, tratamiento físico-químico, decantación primaria, cuba de aireación y decantador secundario. Asimismo, la EDAR alberga un sistema de digestión anaerobia, deshidratación mecánica centrífuga, secado térmico y cogeneración que se enmarca en la Línea de fango.

La EDAR Font de la Pedra (Muro) establece un proceso biológico de aireación prolongada mediante un procedimiento orbal enmarcado en la Línea de agua. Este proceso consta de los ciclos de pretratamiento, tanque de homogeneización, reactor de aireación, decantación secundaria, filtración y desinfección mediante ultravioletas. Además, la Línea de fango establece un sistema de espesador y deshidratación centrífuga. 
Cuadro 1

ESTACIONES EDAR SEGÚN EL MUNICIPIO Y EL CAUDAL, $2006\left(\mathrm{~m}^{3} / \mathrm{dia}\right)$

\begin{tabular}{|c|c|c|c|}
\hline EDAR & Caudal (M³/día) & EDAR & Caudal (M³/día) \\
\hline Alcoi & 24.000 & Alqueria d'Asnar & Vierte a Muro \\
\hline Banyeres de Mariola & 2.500 & Balones & 72 \\
\hline Beneixama & Vierte a Villena & Benasau & 60 \\
\hline Benifallim & 75 & Beniarrés & 400 \\
\hline Biar & 1.500 & Benilloba & 400 \\
\hline Camp de Mirra & Vierte a Villena & Benillup & 38 \\
\hline Canyada & Vierte a Villena & Benimarfull & 100 \\
\hline Penàguila & 200 & Benimassot & 72 \\
\hline Total Alcoià & 28.275 & Cocentaina & Vierte a Muro \\
\hline Castalla & 4.000 & Fageca & 100 \\
\hline Ibi & 6.000 & Famorca & 60 \\
\hline Onil & Vierte a Castalla & Gaianes & 230 \\
\hline Tibi & 180 & Gorga & 72 \\
\hline Total Foia de Castalla & 10.180 & l'Orxa & 180 \\
\hline Agres & 220 & Millena & 72 \\
\hline Alcocer & 60 & Muro & 15.000 \\
\hline Alcoleja & 100 & Planes & 180 \\
\hline Alfafara & 160 & Quatretondeta & 75 \\
\hline Almudaina & 60 & Tollos & 38 \\
\hline & & Total El Comtat & $\mathbf{1 7 . 7 4 9}$ \\
\hline
\end{tabular}

FUENTE: EPSAR (2008).

La EDAR Foia de Castalla (Castalla) incluye una Línea de agua a través de la que se desarrolla un tratamiento físico-químico y biológico mediante fangos activados y la eliminación de nutrientes mediante las fases de pretratamiento, tratamiento físico-químico, decantación primaria, cuba de aireación, decantador secundario, filtración y desinfección. También desarrolla un sistema de digestión aerobia y deshidratación mecánica centrífuga dentro de la Línea de fango.

La EDAR de Ibi ha implantado un proceso físico-químico y biológico mediante fangos activados y de eliminación de nutrientes dentro de la Línea de agua. Este proceso consta de las etapas de pretratamiento, tanque de homogeneización, físico-químico, decantación primaria, cuba de aireación, decantador secundario y desinfección. Asimismo, ha puesto en marcha un tratamiento mediante estabilización con cal, deshidratación mecánica con filtros de banda y secado térmico dentro de la Línea de fango.

La EDAR de Villena desarrolla un proceso biológico de lagunaje aireado dentro de la Línea de agua que se articula en las fases de pretratamiento, lagunas aireadas, decantación secundaria y laguna de maduración. La Línea de fango establece el desarrollo de un tratamiento de espesador y deshidratación mecánica con filtro de banda. 
Figura 3. Estación EDAR Font de la Pedra de Muro

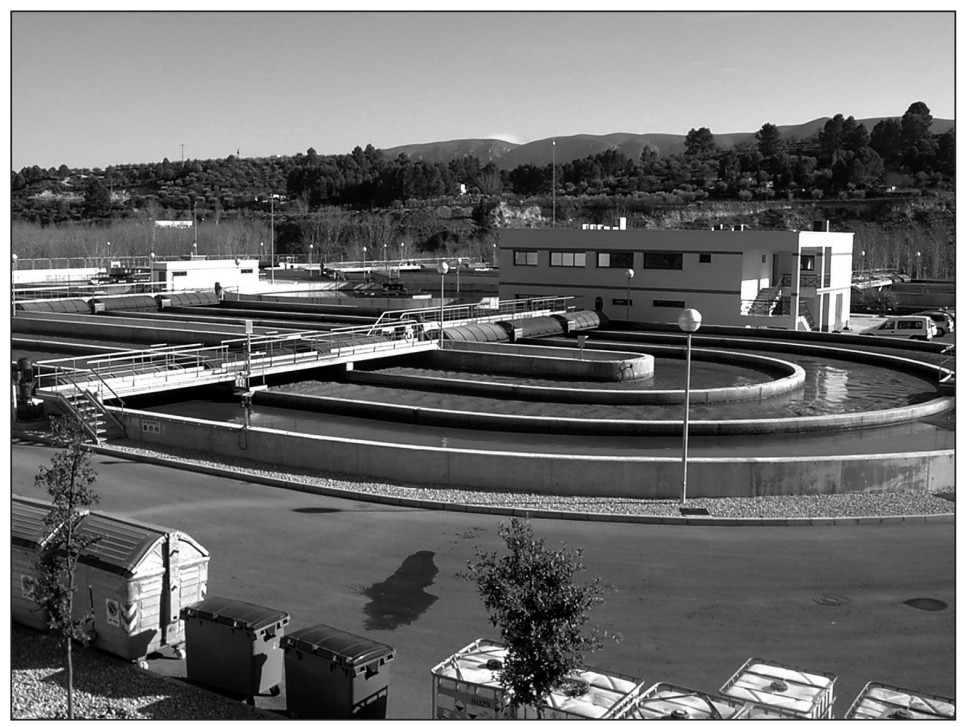

FUENTE: J. Tormo (2005).

FIgURA 4. Estación EDAR de Millena

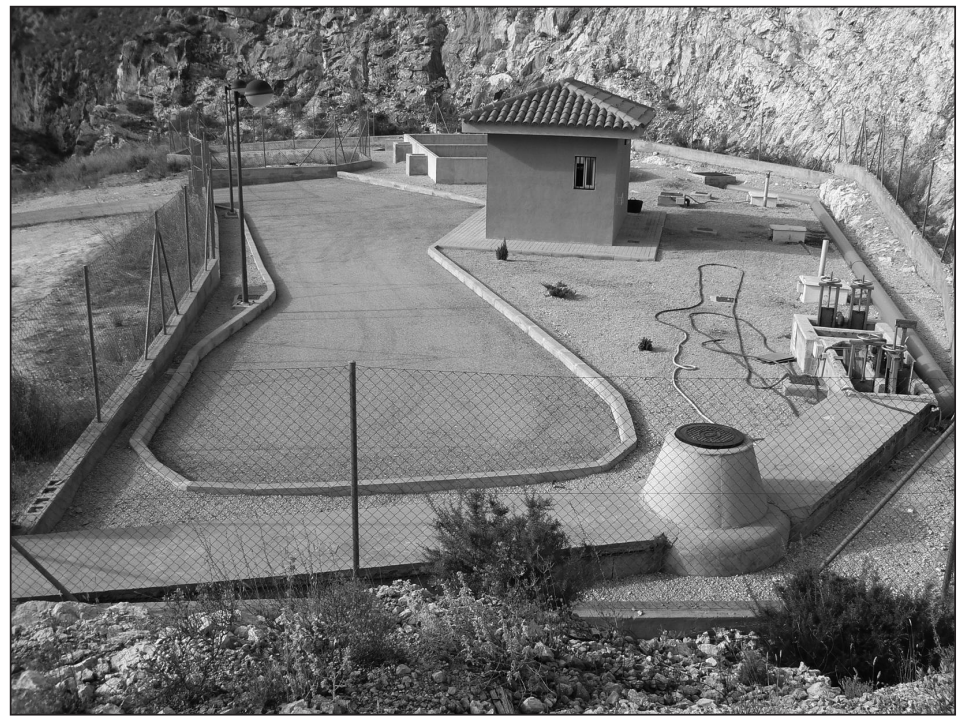

FUENTE: J. Tormo (2008). 
Cuadro 2

PRINCIPALES ESTACIONES DE DEPURACIÓN EDAR SEGÚN EL CAUDAL $Y$ LA POTENCIA, $2006\left(\mathrm{~m}^{3} /\right.$ dia y $\left.\mathrm{kw}\right)$

\begin{tabular}{|c|c|c|c|}
\hline EDAR & Caudal (m³/día) & Potencia (kw) & Tratamiento \\
\hline Els Algars (Alcoi) & 24.000 & 775 & $\begin{array}{l}\text { Línea de agua } \\
\text { Línea de fango }\end{array}$ \\
\hline Font de la Pedra (Muro) & 15.000 & 1.224 & $\begin{array}{l}\text { Línea de agua } \\
\text { Línea de fango }\end{array}$ \\
\hline Foia de Castalla (Castalla) & 4.000 & 516 & $\begin{array}{l}\text { Línea de agua } \\
\text { Línea de fango }\end{array}$ \\
\hline Ibi & 6.000 & 600 & $\begin{array}{l}\text { Línea de agua } \\
\text { Línea de fango }\end{array}$ \\
\hline Villena & 8.000 & 275 & $\begin{array}{l}\text { Línea de agua } \\
\text { Línea de fango }\end{array}$ \\
\hline
\end{tabular}

FUENTE: EPSAR (2008).

Las comarcas de l'Alcoià, la Foia de Castalla y el Comtat disponen de dos de las tres plantas de secado térmico existentes en el espacio valenciano. La EDAR Els Algars (Alcoi) incluye una planta de secado térmico con capacidad para tratar $1.000 \mathrm{~kg}$ de materia húmeda a la hora y cuenta con una capacidad de evaporación de 600 1/h. La construcción de esta planta de secado térmico de fangos y cogeneración de la EDAR de Alcoi finalizó en 2005. La inversión realizada ha sido de 3,06 millones de euros. Por su parte, la planta de secado ubicada en la EDAR de Ibi cuenta con una capacidad de proceso de $700 \mathrm{~kg}$ de materia húmeda a la hora y con una capacidad de evaporación de 500 1/h.

Por último, se debe indicar que EPSAR ha invertido un total de 35,11 millones de euros entre 2004 y 2007 en las comarcas de l'Alcoià, la Foia de Castalla y el Comtat. Esta partida se ha destinado a ejecutar las siguientes obras:

- Mejora de la EDAR Font de la Pedra de Muro (15,24 millones de euros).

- Modificación del proceso de eliminación de nutrientes de la EDAR Foia de Castalla (2,34 millones de euros).

- Depósito y red de distribución de uso industrial en Alqueria d'Asnar (961.345 euros).

- Depósitos y red de distribución industrial en Muro (1,37 millones de euros).

- Tratamiento terciario para uso industrial en la planta Els Algars de Alcoi (15,20 millones de euros).

\subsection{La gestión mancomunada de los recursos hídricos}

\subsubsection{La Mancomunidad de Municipios Font de la Pedra}

Durante el año 2005, los municipios de Alqueria d'Asnar, Cocentaina y Muro pusieron en marcha varias iniciativas con las que pretendían impulsar la depuración de las aguas residuales y su posterior utilización, siendo necesario para ello adecuar los servicios de las depuradoras Els Algars y Font de la Pedra. Algunas de ellas no se ejecutaron por la negativa de varias administraciones públicas. 
A partir de todas estas actuaciones y propuestas, los ayuntamientos de Alqueria d'Asnar, Cocentaina y Muro concretaron un gran acuerdo inicial ${ }^{3}$ a finales de 2005 que sirvió para la constitución de la Mancomunidad de Municipios de la Font de la Pedra a mediados de $2006^{4}$.

La aprobación definitiva de los estatutos de la entidad supuso la puesta en marcha de la mancomunidad. Entre sus principales objetivos están los siguientes:

- El control de los vertidos en las redes municipales de alcantarillado.

- El saneamiento, la depuración y la reutilización de las aguas residuales.

- Otros servicios incluidos en el ámbito de la competencia municipal según los términos de la legislación del estado y de la comunidad autónoma.

Las actuaciones de la Mancomunidad han tomado como soporte la aplicación del Plan terciario de uso y utilización del agua depurada de la planta Font de la Pedra, que se ubica en el término municipal de Muro, dentro de la pedanía de Benàmer, aguas debajo de los tres municipios. En este sentido, destacan las siguientes actuaciones:

- Construcción de tres depósitos de agua con un total de $15.000 \mathrm{~m}^{3}$ para el abastecimiento de los municipios y empresas de la zona, dos de ellos en Muro (12.000 $\left.\mathrm{m}^{3}\right)$ y uno en Alqueria d'Asnar $\left(3.000 \mathrm{~m}^{3}\right)$.

- Construcción de las estaciones de bombeo y de la red de distribución del agua entre los depósitos, los municipios y las empresas.

Ambos proyectos contaron con una partida total aproximada de 1,40 millones euros. Uno de sus principales beneficios es que el agua pueda ser utilizada en la industria, la extinción de incendios y la limpieza de calles y viales.

\subsubsection{La Mancomunidad Intermunicipal de la Foia de Castalla}

Los ayuntamientos de Onil y Castalla acordaron en 1986 aprobar $^{5}$ los estatutos y la constitución de la Mancomunidad Intermunicipal de la Foia de Castalla, de la que ambos formaban parte. Su sede se estableció de forma alternativa entre ambos municipios.

Esta mancomunidad de municipios tiene como objeto gestionar el control, la depuración y la reutilización de las aguas residuales de ambos municipios a través de la implementación de actuaciones que permitan mejorar la función de la estación de depuración de aguas residuales de Castalla. Se trata de una instalación que cuenta con una capacidad de depuración de aguas residuales de $4.000 \mathrm{~m}^{3} /$ día y a la que vierten sus aguas residuales los municipios de Castalla y Onil.

\subsubsection{La Mancomunidad de Servicios de Beneixama, Camp de Mirra y Canyada}

Los municipios de Beneixama, Camp de Mirra y Canyada constituyeron en el año 2000 la Mancomunidad de Servicios de Beneixama, Camp de Mirra y Canyada. Según

3 El anteproyecto de los estatutos de la mancomunidad fue aprobado por una asamblea formada por los concejales de los tres ayuntamientos el día 4 de octubre de 2005 y se hizo público en el BOP número 261 de 15 de noviembre de 2005 .

4 Orden de 12 de junio de 2006, por la que se Homologan los Estatutos de la Mancomunitat Font de la Pedra (DOGV 5.294 de 04/07/2006).

5 Aprobados por la Orden de 10 de junio de 1986, sobre la Constitución y Estatutos de la Mancomunitat de la Foia de Castalla (DOGV 488 de 17/12/1986). 
sus estatutos ${ }^{6}$, el objetivo de la mancomunidad es prestar los servicios sociales necesarios a la población de los tres municipios mancomunados, aunque indica que también puede atender la prestación de otros servicios siempre que los plenos de los ayuntamientos lo aprueben por mayoría absoluta.

En el transcurso de los cinco años siguientes los municipios mancomunados llegaron a diversos acuerdos que les han permitido modificar sus estatutos ${ }^{7}$ en 2005 y atribuir a la mancomunidad las competencias que la convierten en la entidad básica para el desarrollo integral de sus municipios.

Según la modificación y aprobación de sus nuevos estatutos, la Mancomunidad de Servicios de Beneixama, Camp de Mirra y Canyada gestiona en la actualidad una serie de servicios entre los que se encuentra la prestación de los servicios de abastecimiento de agua.

\section{La reutilización y el abastecimiento de agua potable a los municipios y las indus- trias. El caso de la comarca El Comtat}

\subsection{La importancia del agua en el desarrollo social y económico}

La existencia de una red de abastecimiento de agua potable en buenas condiciones es un factor clave para el desarrollo del bienestar social, la mejora de la calidad de vida de la población y la promoción económica de un espacio geográfico determinado.

El ser humano ha mantenido una lucha constante por aprovisionarse de agua con el objeto de cubrir sus necesidades vitales y las derivadas de las actividades económicas a lo largo del tiempo. Estas necesidades han cambiado en relación a la evolución de la tecnología y la mejora de la calidad (Salvà, 1998), por lo que el agua se ha convertido en un bien de doble funcionalidad. Por una parte, se trata de un bien social que sirve para su desarrollo personal y, por otra parte, se trata de un bien económico utilizado en las actividades productivas. Sin embargo, según el Atlas Le Monde Diplomatique (2007), el acceso al agua no está garantizado para todos los habitantes del mundo a pesar de los compromisos adoptados por todos los países. En concreto, más de 1.100 millones de personas carecen de agua potable y 2.400 millones no cuentan con instalaciones sanitarias adecuadas para su abastecimiento. Según V. Peñas (2006, p. 13), el agua es «un don de la naturaleza que posibilita la vida de todos los seres vivos del planeta. Sin agua no hay vida, y sin embargo en los albores del siglo XXI la humanidad continúa degradando los ecosistemas acuáticos, poniendo en grave peligro nuestra propia supervivencia económica y personal».

Las comarcas de l'Alcoià, la Foia de Castalla y el Comtat forman parte de la Cuenca del Xúquer. Ésta se organiza en nueve sistemas de explotación entre los que se encuentran las áreas Serpis y Vinalopó-Alacantí, es decir, en las que se ubican los municipios del interior septentrional de Alicante.

Según Gil y Rico (2008), la Cuenca del Xúquer cuenta con unos recursos anuales de $4.000 \mathrm{hm}^{3}$, siendo la disponibilidad real de agua del 75\%. En su opinión, se trata de una cuenca deficitaria que ha sufrido durante los últimos años la sobreexplotación de sus acuíferos. En concreto, el sistema de explotación Serpis registra un déficit anual de 24,5 hm y el sistema Vinalopó-Alicantí un déficit de $176,1 \mathrm{hm}^{3}$.

6 Orden de 11 de abril de 2000, por la que se Homologan los Estatutos de la Mancomunidad de Servicios Beneixama, Campo de Mirra y Cañada (DOGV 3.761 de 31/05/2000).

7 Orden de 23 de mayo de 2005, por la que se Homologa la Modificación de los Estatutos de la Mancomunitat de Serveis de Beneixama, Camp de Mirra i Canyada (DOGV 5.025 de 14/06/2005). 
Entre las iniciativas puestas en marcha para mejorar la gestión de los recursos hídricos en las comarcas de l'Alcoià, la Foia de Castalla y el Comtat se debe destacar el Plan estratégico para la gestión eficiente del agua (2004-2007) de Alcoi, que ha logrado reducir en 177 millones de litros el consumo anual de agua en el municipio. Para ello, ha sido necesario establecer una partida específica de cuatro millones de euros que se han destinado a la revisión de $523 \mathrm{~km}$ de tuberías, sustituyéndose 22 de ellos, la reparación de 127 fugas, la instalación de 26.738 contadores y a la edición de material educativo y de difusión del plan. Otra iniciativa interesante ha sido la desarrollada en el municipio de Muro. El Ajuntament de Muro ha reparado y renovado la red de conducciones de agua potable del término municipal durante el año 2007 tras la obtención de financiación por parte de la Diputació d'Alacant

y de la Conselleria d'Infraestructures i Transports. Esta iniciativa ha permitido reducir las pérdidas de agua registradas durante los últimos años, ya que ha pasado de perder del $80 \%$ del agua extraída de los pozos en 1999 a menos del 8\% en la actualidad.

\subsection{La Junta de Usuarios del Acuífero de la Sierra de Mariola}

En diciembre de 2001 se constituyó la Comunidad del Acuífero de la Sierra de Mariola, que está formada por 40 usuarios del acuífero de Mariola, que abastece a los municipios de Cocentaina, Muro, Alqueria d'Asnar y Biar, entre otros.

Este organismo se reunió en asamblea por primera vez en el municipio de Cocentaina con el objeto de constituir una comunidad de usuarios a través de la redacción de sus estatutos y de la realización de varios trámites administrativos. Tiene entre sus antecedentes básicos la Taula de l'Aigua, entidad que agrupó a los partidos políticos, los grupos ecologistas, los regantes y los empresarios de Cocentaina y Muro con el fin de fomentar la explotación respetuosa de los recursos hídricos de la zona.

Uno de sus primeros acuerdos se firmó en febrero de 2002 y estableció que los ayuntamientos controlasen el 55\% de las extracciones y el $45 \%$ restante se regulase de acuerdo a las concesiones que se atribuyeran a las industrias y los particulares, entre otros.

Las extracciones realizadas de forma continua en los acuíferos de Mariola durante los últimos años han tenido como consecuencia su sobreexplotación, es decir, que el volumen de aguas extraído supera al volumen de agua que reciben anualmente. Esta situación se ha visto agravada por parte de las empresas y los particulares que realizan extracciones ilegales y diezman así el caudal de los acuíferos. Por este motivo, la Junta de Usuarios decidió implementar diversas actuaciones que permitiesen reducir el volumen de agua extraída a través de la instalación de controladores en los pozos de la comunidad y de la mejora de la red de distribución para evitar las pérdidas sufridas por la infiltración.

La Junta de Usuarios se reunió en junio de 2005 en asamblea general con el objeto de aprobar los presupuestos de la entidad. El presupuesto anual de la Junta ascendió ese año a 12.000 euros, que fueron sufragados al $50 \%$ por los ayuntamientos y las empresas industriales de la zona. Esta reunión sirvió para que la Junta elaborase siete propuestas de actuaciones a realizar con las que conseguir reducir las extracciones, así como mejorar la distribución y la utilización de los recursos. Entre estos acuerdos, se debe destacar que la Junta de Usuarios solicitó a la Conselleria d'Agricultura, Pesca i Alimentació y a la Diputació d'Alacant la transferencia de los pozos del acuífero para la entidad. Con esta actuación se pretendía lograr el control sobre el acuífero y poder actuar fiscalizando las extracciones y estableciendo límites en su gestión. El objeto final era consensuar con los usuarios las medidas a realizar y los volúmenes máximos a extraer. Asimismo, se pretendía conseguir que la propia Junta de Usuarios fuera el principal órgano encargado de la utilización de los recursos de la zona. 


\subsection{Los problemas detectados y las actuaciones ejecutadas para la mejora del abasteci- miento municipal. El caso de la comarca el Comtat}

Como se ha indicado arriba, las distintas administraciones públicas han ejecutado diversas actuaciones durante los últimos años a fin de mejorar la gestión y la utilización de los recursos hídricos en la comarca del Comtat. Éstas han tenido como objeto superar una serie de problemas que se detectaron en muchos municipios durante los primeros años del siglo XXI a causa de las malas condiciones de las redes de abastecimiento de los municipios. Estas situaciones quedaron reflejadas en las noticias publicadas por el periódico Ciudad de Alcoy:

- «Un reventón en la zona de Les Fontanelletes deja a Muro sin agua» (02/09/2000).

- «Benasau. Diputación subvenciona la reparación de la infraestructura hidráulica» $(18 / 10 / 2000)$.

- «Gaianes. Diputación invierte más de 1,5 millones en la red de aguas» (02/12/2000).

- «Más de 300 millones para saneamiento de aguas residuales en el Comtat» $(07 / 12 / 2000)$.

- «Inversiones en Benilloba y Beniarrés para mejorar infraestructuras hidráulicas y comerciales» (12/12/2000).

- «Benilloba. La renovación de la red de saneamiento costará 35 millones» (20/12/2000).

- «Gorga. Una avería en la bomba deja a los vecinos sin agua potable» $(07 / 01 / 2001)$.

- «Gorga. El arreglo de la bomba de agua cuesta 1.300 .000 pesetas» (11/01/2001).

Algunos municipios de la comarca como son Benasau, Benilloba, Beniarrés, Gaianes, Gorga y Muro tuvieron importantes problemas de abastecimiento hídrico a partir del año 2000. Por esta razón, la Diputació d'Alacant ha financiado durante los últimos años numerosa obras e instalaciones a través de diferentes planes de actuación. En este sentido, se deben destacar las siguientes actuaciones realizadas a través del Plan Complementario de Obras y Servicios (PCOS) y del Plan Operativo Local (POL):

- Reposición del ramal de abastecimiento de agua potable del manantial al depósito regulador de Balones (PCOS, 72.075 euros).

- Renovación de la conducción de agua potable desde el manantial al municipio de Quatretondeta (PCOS, 120.202 euros).

- Instalación de un depósito regulador de aguas en Fageca (POL, 209.000 euros).

- Renovación parcial de la red de agua potable de Gaianes (POL, 140.000 euros).

- Instalación de un pozo de reserva de agua potable en Beniarrés (POL, 79.000 euros).

- Mejora de las infraestructuras hidráulicas de Balones (POL, 60.000 euros).

- Renovación de la red de alcantarillado de Alcocer (POL, 199.995 euros).

- Mejora de la red de abastecimiento de agua potable de Beniarrés (POL, 225.000 euros).

- Renovación de la red de conducción de agua potable al municipio de Gorga (POL, 144.900 euros).

Además, mediante el Plan Hidráulico Provincial de la Diputació d'Alacant se han realizado las siguientes infraestructuras:

- Instalación un depósito de $1.000 \mathrm{~m}^{3}$ en Benimarfull.

- Conexión de las aguas residuales de Benilloba a la red de saneamiento. 
- Adecuación de la red y de los colectores fluviales de Planes.

- Construcción de un depósito de agua en la zona de La Queola en Cocentaina.

- Renovación del alcantarillado de Benasau.

- Reparación de varios desperfectos en la bomba de agua que abastecen a Benasau.

\subsection{Proyectos para la utilización de aguas depuradas. El caso de la Mancomunidad de Municipios de la Font de la Pedra}

Los municipios de Alqueria d'Asnar, Cocentaina y Muro apostaron por favorecer la depuración de las aguas residuales y su utilización posterior durante el año 2005. Los tres ayuntamientos solicitaron a la Conselleria d'Infraestructures i Transports la realización de las obras que permitiesen a los municipios y a las industrias de sus términos acceder a los recursos de agua depurada a principios de 2005. Requerían que se aprobara la realización de infraestructuras que permitiesen el transporte de agua depurada desde la depuradora Els Algars hasta el municipio de Cocentaina y desde la depuradora Font de la Pedra en Benàmer (pedanía del municipio de Muro) hasta los núcleos de Muro y Alqueria d'Asnar.

El Ministerio de Medio Ambiente incluyó como enmiendas al Plan Hidrológico Nacional la realización de actuaciones para mejorar la utilización de las aguas residuales. El gobierno central tenía que llevar a cabo una serie de actuaciones que permitiesen a los ayuntamientos de Alcoi, Alqueria d'Asnar, Cocentaina y Muro utilizar 3,50 hm $\mathrm{hm}^{3}$ de agua de la depuradora Els Algars. Para ello, se aprobó un plan de actuación con un presupuesto de 6 millones de euros y con un plazo de ejecución de un año. Sin embargo, la Conselleria d'Infraestructures i Transports decidió doblar la capacidad de depuración de la estación Els Algars en septiembre de 2005 y utilizar un porcentaje importante de sus aguas en el proyecto de campo de golf y urbanización Xirillent en Alcoi, mientras que las intenciones planteadas por los ayuntamientos de Alqueria d'Asnar, Cocentaina y Muro eran incrementar los recursos de la depuradora con el objeto de proporcionar un caudal suficiente para su reutilización en la industria, en la limpieza urbana y en el mantenimiento de los parques y los jardines.

Ante esta situación, el Ajuntament de Muro llegó a un acuerdo con EPSAR para la construcción de tres depósitos de aguas depuradas provenientes de la depuradora Font de la Pedra. Además, EPSAR hizo público en noviembre de 2005 el proyecto de instalación de un depósito para el almacenamiento de agua depurada para el uso municipal en la limpieza viaria, el riego y la industria en el polígono industrial La Llaona (Cocentaina). Éste proyecto tenía un presupuesto de 15 millones de euros y permitiría obtener $15.000 \mathrm{~m}^{3}$ diarios de agua.

Estas actuaciones favorecieron que los ayuntamientos de Alqueria d'Asnar, Cocentaina y Muro firmasen diversos acuerdos durante el año 2006 que promovieron la constitución de la Mancomunidad de Municipios de la Font de la Pedra.

La prensa local no se mantuvo ajena a este acuerdo y siguió de cerca el proceso de constitución. De este modo, el periódico Ciudad de Alcoy registró, entre otras, las siguientes noticias:

- «Luz verde a la mancomunidad de servicios Font de la Pedra» (08/07/2006).

- «La Mancomunitat de la Font de la Pedra inicia su andadura» (10/08/2006).

- «Alqueria d'Asnar. Se aprovecharán las aguas residuales de la depuradora» $(16 / 09 / 2006)$.

- «Adjudican las obras para la utilización de agua depurada» (30/09/2006).

- «Iniciada la conexión de la depuradora a las empresas» (24/10/2006). 
La aprobación de los estatutos de la entidad supuso la puesta en marcha definitiva de la mancomunidad, por ello, los alcaldes de los municipios realizaron una serie de declaraciones al respecto. En concreto, J. Marset, por entonces alcalde de Cocentaina, afirmaba que «este proyecto supondrá un desahogo para el acuífero de Mariola». Además, según J. Pascual, alcalde de Alqueria d'Asnar, el proyecto favorece «el uso del agua en las empresas, la lucha contra la falta de agua y que las industrias no utilicen agua de consumo humano» ${ }^{8}$.

Las actuaciones de la mancomunidad se enmarcan dentro del Plan terciario de uso y utilización del agua depurada de la instalación Font de la Pedra, que articula las iniciativas desarrolladas y las obras que se ejecutan para la prestación de los servicios. Entre ellas, se deben destacar la construcción de tres depósitos de agua de un total de $15.000 \mathrm{~m}^{3}$ para el abastecimiento a los municipios y empresas de la zona, de los que dos se localizan en Muro $\left(12.000 \mathrm{~m}^{3}\right)$ y uno en Alqueria d'Asnar $\left(3.000 \mathrm{~m}^{3}\right)$, así como la construcción de las estaciones de bombeo y de la red de distribución del agua entre los depósitos, los municipios y las empresas. Este proyecto ha contado con un presupuesto aproximado de 140 millones euros y ha permitido la utilización del agua depurada a partir de mayo de 2007 en el uso industrial, el uso en la extinción de incendios y la limpieza de calles y viales ${ }^{9}$.

Por último, se debe indicar que los tres ayuntamientos han redactado y aprobado una Ordenanza para controlar los vertidos industriales en la depuradora en 2008. Esta normativa ha entrado en funcionamiento a partir de su publicación en el DOCV en junio de 2008 y regula los niveles de contaminación permitidos en el desagüe por parte de las empresas, así como las sanciones que se imponen a las empresas de los municipios al incumplir la ordenanza. Además, establece que las industrias deberán disponer de autorización municipal para verter a la red y están obligadas a que se realicen revisiones periódicas sobre la calidad de las aguas vertidas.

\subsection{El embalse de Beniarrés: actuaciones y mejoras realizadas}

El embalse de Beniarrés se empezó a construir en el año 1945, aunque su consolidación definitiva como infraestructura hidrográfica y sus inicios oficiales de funcionamiento datan de 1958. El embalse de Beniarrés tiene una capacidad de almacenamiento de $26 \mathrm{hm}^{3}$ y ocupa una superficie total de 268 hectáreas, estando su capacidad ocupada aproximadamente al $50 \%$ como media, según los datos de la Confederació Hidrogràfica del Xúquer (2007), si bien las lluvias acaecidas durante 2008 han situado su ocupación en el $90 \%$.

En el año 2002 se invirtieron más de 1,50 millones de euros para la consolidación del embalse de Beniarrés. Se trata de una serie de obras que se desarrollarán hasta el año 2010 y que cuentan con un presupuesto total superior a los 17 millones de euros, cuyo objeto es consolidar la presa, impermeabilizarla para evitar fugas y poner en marcha un nuevo sistema de drenaje con lo que se permitirá incrementar su capacidad en un $40 \%$.

Los equipos de la Confederació Hidrogràfica del Xúquer han realizado diversas obras en el embalse con el objeto de prevenir y conservar esta infraestructura evitando el peligro de fugas desde principios de 2005. Para ello, se han llevado a cabo varias inyecciones de cemento en los cimientos de la presa del embalse a través de un sistema de galerías con el

8 «Luz verde a la mancomunidad de servicios Font de la Pedra», en periódico Ciudad de Alcoy (08-072006).

9 La entidad EPSAR está ejecutando en la actualidad las obras de adaptación terciaria de la EDAR Els Algars y la construcción de la red de canalizaciones que permitirán la reutilización de aguas depuradas por parte de las industrias de los municipios de Alcoi y Cocentaina. Este proyecto cuenta con un presupuesto de 15 millones de euros, aproximadamente, que se destinarán a la construcción de dos estaciones de bombeo de agua y de dos depósitos de distribución que permitirán la reutilización de $15.000 \mathrm{~m}^{3}$ de agua al día. 
que se han consolidado sus cimientos y se ha impermeabilizado la presa. En total, se han aplicado 540 toneladas de cemento. En 2005 se procedió al sellado de una fuga detectada en una de las laderas del embalse. Esta obra contó con un presupuesto de 61.747 euros y consistió en la delimitación y consolidación de la zona. Para estas obras se contó con la financiación del Ministerio de Medio Ambiente y de la propia confederación hidrográfica.

Dos años después, a lo largo del 2007, se avivó la polémica sobre la calidad ambiental de las aguas del embalse. En el mes de abril, el Ajuntament de Beniarrés hizo pública su intención de denunciar a la Confederació Hidrogràfica del Xúquer por su no implicación en la mejora de la calidad de las aguas y por no atender a la solicitud del ayuntamiento de realizar una auditoria ambiental del embalse. Dos meses después, el Ajuntament de Beniarrés presentó ante el Síndic de Greuges una denuncia por estos motivos. Con todo, el 1 de junio de 2005, la Confederació Hidrogràfica del Xúquer y los ayuntamientos con mayor implicación en el embalse, es decir, Alcoi, Cocentaina, Alqueria d'Asnar, Benimarfull, Muro, Gaianes, Planes y Beniarrés, acordaron la realización de una auditoria ambiental del embalse. Tras varios estudios, se detectó que la calidad de las aguas vertidas al embalse se encontraba en niveles muy bajos.

Estos informes sirvieron para determinar la posición de los ayuntamientos implicados en torno a dos cuestiones de vital importancia para la recuperación de la calidad de las aguas del embalse como son la implantación del sistema de depuración terciaria en las depuradoras y la limitación de los proyectos de urbanizaciones en torno al embalse. En este sentido, con el objeto de proceder a la limpieza y depuración de las aguas del embalse es necesario poner en marcha el sistema de depuración terciario en la planta depuradora de Els Algars (Alcoi), ya que en la planta Font de la Pedra (Muro) ya se encuentra operativo. Además, es necesario revisar los proyectos de urbanizaciones que se han proyectado en el perímetro del pantano dentro del término municipal de Planes, ya que pueden suponer un fuerte impacto para el paisaje y una sobrecarga importante para la zona.

\section{Conclusiones}

En el espacio geográfico constituido por las comarcas alicantinas de l'Alcoià, la Foia de Castalla y el Comtat se han puesto en marcha durante los últimos años una serie de actuaciones que han permitido mejorar el abastecimiento de agua potable a los municipios y las industrias a partir de la depuración y reutilización de las aguas residuales mediante infraestructuras como son las estaciones de depuración de aguas residuales (EDAR), las plantas de secado térmico y la renovación de las infraestructuras de abastecimiento y distribución.

Estas actuaciones se han implementado principalmente mediante las iniciativas de la Generalitat Valenciana a través de la Entitat Pública de Sanejament d'Aigües Residuals (EPSAR), la Diputació d'Alacant, las actuaciones municipales y la colaboración municipal a través de las mancomunidades de municipios y han supuesto una mejora sustancial en la gestión de los recursos hídricos que puede tomarse como ejemplo a la hora de poner en marcha acciones de este tipo en otras áreas del espacio valenciano.

\section{Bibliografía}

BAO, M. (2001): «Tratamiento avanzado de efluentes industriales», en El agua, un bien para todos. Conservación, recuperación y usos. Salamanca, Universidad de Salamanca, p. 181-198.

BEÜT, E. (1980): Geografia elemental del Regne de València. València, Marí Montañana, 110 pp. EPSAR (2008): Estadísticas de las infraestructuras hídricas en la Comunidad Valenciana. Disponible en www.gva.es 
GIL, A.; RICO, A. (2008): Políticas del agua III. Murcia, ESAMUR, 484 p.

HERNÁNDEZ, A. (2007): Saneamiento y alcantarillado. Vertidos residuales. Madrid, Colegio de Ingenieros de Caminos, Canales y Puertos, $1045 \mathrm{p}$.

INSTITUTO NACIONAL DE ESTADÍSTICA (2007): Revisión del Padrón Municipal de habitantes 2006. Disponible en www.ine.es/inebase/cgi/axi.htm

MATEU, F. (1933): El País Valencià. València, L'Estel, 133 pp.

NAREDO, J. M. (2006): «La encrucijada de la gestión del agua en España», en El agua en el siglo XXI. Gestión y planificación. Zaragoza, Atlas, pp. 2-28.

PEÑAS, V. (2006): ¿Llueve a gusto de todos?. Madrid, Espasa, 169 p.

PROMOCIONS CULTURALS SA (1970): Nomenclàtor geogràfic del País Valencià. València, Promocions Culturals SA, 34 pp.

OBSERVATORIO OCUPACIONAL DE LA PROVINCIA DE ALICANTE (2007): Informe del mercado de trabajo de la provincia de Alicante. Formato Cd Rom.

SALVÀ, P. (1998): «L'activitat humana i la contaminació de les aigües», en L'Aigua i la vida. La seva utilització. Palma de Mallorca, Fundació La Caixa - Universitat de les Illes Balears, pp. 161-198.

SANCHÍS, M. (1966): Contribució al nomenclàtor geogràfic del País Valencià. Barcelona, Institut d'Estudis Catalans, $24 \mathrm{pp}$.

TORMO, J. (2006): El Comtat. Diagnòstic territorial i estratègies de futur. Muro, Ajuntament de Muro, 208, pp.

TORMO, J. (2008): «La mancomunidades de municipios como instrumentos para la participación y el desarrollo local. El caso de la comarca el Comtat», en Sostenibilidad en los espacios rurales. Alicante, Ministerio de Agricultura, Generalitat Valenciana y Universitat d'Alacant, pp. 280-290.

VV.AA. (2007): Atlas Le Monde Diplomatique. València, Ed. Cibermonde, 194 pp. 